\title{
Snowflake-Shaped ZnO Nanostructures-Based Gas Sensor for Sensitive Detection of Volatile Organic Compounds
}

\author{
Tianli Han, ${ }^{1,2}$ Xuexue Li, ${ }^{3}$ Xiaoman Zhang, ${ }^{4}$ Jinyun Liu, ${ }^{4}$ and Jinjin Li ${ }^{1}$ \\ ${ }^{1}$ Key Laboratory for Thin Film and Microfabrication of the Ministry of Education, Department of Micro-/Nano-Electronics, \\ Shanghai Jiao Tong University, Shanghai 200240, China \\ ${ }^{2}$ College of Chemistry and Material Engineering, Chaohu University, Chaohu, Anhui 238000, China \\ ${ }^{3}$ Key Laboratory of Functional Molecular Solids, Ministry of Education, Center for Nano Science and Technology, \\ College of Chemistry and Materials Science, Anhui Normal University, Wuhu 241000, China \\ ${ }^{4}$ Nanomaterials and Environment Detection Laboratory, Institute of Intelligent Machines, Chinese Academy of Sciences, \\ Hefei, Anhui 230031, China
}

Correspondence should be addressed to Jinjin Li; lijinjin.physics@gmail.com

Received 4 October 2016; Accepted 15 January 2017; Published 7 February 2017

Academic Editor: Veer P. S. Awana

Copyright (C) 2017 Tianli Han et al. This is an open access article distributed under the Creative Commons Attribution License, which permits unrestricted use, distribution, and reproduction in any medium, provided the original work is properly cited.

\begin{abstract}
Volatile organic compounds (VOCs) have been considered severe risks to human health. Gas sensors for the sensitive detection of VOCs are highly required. However, the preparation of gas-sensing materials with a high gas diffusion performance remains a great challenge. Here, through a simple hydrothermal method accompanied with a subsequent thermal treatment, a special porous snowflake-shaped $\mathrm{ZnO}$ nanostructure was presented for sensitive detection of VOCs including diethyl ether, methylbenzene, and ethanol. The fabricated gas sensors exhibit a good sensing performance including high responses to VOCs and a short response/recovery time. The responses of the $\mathrm{ZnO}$-based gas sensor to $100 \mathrm{ppm}$ ethanol, methylbenzene, and diethyl ether are about 27,21 , and 11, respectively, while the response times to diethyl ether and methylbenzene are less than 10 seconds. The gas adsorptiondesorption kinetics is also investigated, which shows that the gas-sensing behaviors to different target gases are remarkably different, making it possible for target recognition in practical applications.
\end{abstract}

\section{Introduction}

Volatile organic compounds (VOCs) such as diethyl ether and methylbenzene, which are commonly released from chemical reagents, or even building paints, have been considered severe risks to human health $[1,2]$. Efficient detection of VOCs thus is of great interest. Among many other detection methods, gas sensors-based systems are attractive because of their lowcost, high stability, simple operation procedures, and so forth [3-6]. However, accompanied with some other issues, the relatively poor detection limit and low response/recovery speeds limit the applications of gas sensor-based systems. In order to address these issues, many efforts including developing sensitive gas-sensing materials and optimizing sensor structures have been contributed to this field [710]. For example, nanostructured sensing materials, such as nanotubes, nanowires, and nanoparticles, have been prepared, which were found to have much enhanced sensing performance $[11,12]$. Once the dimensions of the materials are reduced, the overlap and aggregation issues within the thick sensing layer of sensors commonly become severe due to the high surface energy of small particles, reducing the effective contact between sensing sites and target gas molecules. Porous structure has been considered a promising method to improve the diffusion of gases and their contact with sensing sites which locate at deep layer. Commonly, using template is necessary for making a porous structure $[13,14]$, which may introduce additional template removal steps, making the preparation procedures complicated.

Here, we present a porous gas-sensing nanostructure with an overall snowflake morphology prepared through a simple method consisting of hydrothermal growth and subsequent thermal conversion. The prepared porous $\mathrm{ZnO}$ nanosheets grow together forming a layered snowflake structure, which 
provides efficient environment for gas diffusion since the branched sheets could retain sufficient interspaces within the sensing layer, while the high porosity enables efficient gas transportation. In our investigations, the snowflake-shaped $\mathrm{ZnO}$ nanostructures were systemically characterized, and the gas-sensing performance to VOCs including diethyl ether, methylbenzene, and ethanol was also demonstrated.

\section{Experimental}

2.1. Preparation of the Snowflake-Shaped Nanostructures. All chemicals were analytical grade, purchased from Shanghai Chemical Reagents Company, and used without further purification. The snowflake-shaped $\mathrm{ZnO}$ nanostructures were prepared through a simple route containing hydrothermal synthesis and annealing treatment. The hydrothermal growth approach was similar to previous report $[15,16]$ with some modifications here. Typically, $1 \mathrm{mM}$ of $\mathrm{Zn}\left(\mathrm{NO}_{3}\right)_{2}$ and $5 \mathrm{mM}$ of urea were dissolved into $35 \mathrm{~mL}$ of deionized water under constantly magnetic stirring for $1 \mathrm{~h}$. Subsequently, the formed solution was transferred into a Teflon-lined stainless steel autoclave (50 mL capacity), which was sealed and heated in an oven at $120^{\circ} \mathrm{C}$ for $5 \mathrm{~h}$. After cooling to room temperature naturally, the precipitates were collected via centrifuge and washed with ethanol and deionized water, followed by drying at $60^{\circ} \mathrm{C}$ for $8 \mathrm{~h}$. In the subsequent thermal treatment, the samples were heated in air at $450^{\circ} \mathrm{C}$ for $1 \mathrm{~h}$ at a ramp rate of $5^{\circ} \mathrm{C} \min ^{-1}$.

2.2. Physical Characterizations. The prepared samples were characterized using a FEI Sirion 200 field emission scanning electron microscope (FESEM) and Philips X' Pert Pro Xray diffractometer (XRD) with $\mathrm{Cu} \mathrm{K} \alpha$ radiation $(1.5418 \AA$ ). The diffraction peaks of crystalline phases were compared with the standard literatures in Joint Committee on Powder Diffraction Standards (JCPDS). The transmission electron microscope (TEM) observations were performed on a JEOL$2100 \mathrm{~F}$ microscope operated at $200 \mathrm{kV}$ accelerating voltage. Elemental analysis was conducted on an ESCALab MK II Xray photoelectron spectrometer (XPS) using nonmonochromatized Mg Ka X-ray beams as the excitation source. Binding energies were calibrated relative to the C1s peak at $284.6 \mathrm{eV}$. The surface area of the nanostructures was measured on a Coulter Omnisorp 100CX Brunauer-Emmett-Teller (BET) instrument using nitrogen adsorption-desorption.

\subsection{Sensor Fabrication and Gas-Sensing Measurements. For} the fabrication of gas sensors based on the prepared $\mathrm{ZnO}$ nanostructures, first, a certain amount of the $\mathrm{ZnO}$ were dispersed in absolute alcohol by ultrasonication for $20 \mathrm{~min}$. Subsequently, the mixed slurry was rotatingly coated onto the surface of an $\mathrm{Al}_{2} \mathrm{O}_{3}$ tube in which there was a Ni-Cr wire used as heater. Then, the sensors were dried in an oven under vacuum. Before the gas detection, in order to enhance the signal stability of the gas sensors, all sensor samples were aged under working conditions for two days.

The gas-sensing measurements were performed using a computer-controlled gas detecting system in which a
Keithley 6487 picoameter/voltage sourcemeter was used as both current recorder and power source. The gas chamber is equipped with both dry air and target gases inputs which can be alternated for requirement. In gas-sensing tests, typical VOCs including diethyl ether, methylbenzene, and ethanol at a series of concentrations ranging from 10 to $200 \mathrm{ppm}$ were employed as targets, respectively. All gas-sensing detections were performed at the same working temperature of gas sensors $\left(200^{\circ} \mathrm{C}\right)$ and humidity of ambient atmosphere (ca. $60 \% \mathrm{RH})$. The response of gas sensor is defined as

$$
S=\frac{R_{\mathrm{air}}}{R_{\mathrm{gas}}}=\left(\frac{I_{\mathrm{gas}}}{I_{\mathrm{air}}}\right)_{V},
$$

where $R_{\text {air }}$ is the resistance in air, while $R_{\text {gas }}$ is that in the gas mixture of pure air and target gas. In terms of Ohm's Law, $S$ can be presented by the current in pure air $\left(I_{\text {air }}\right)$ and in gas mixture $\left(I_{\text {gas }}\right)$ at a constant measuring voltage. Moreover, the response and recovery times were determined by the times for sensor to achieve $90 \%$ of the total resistance change in the cases of gas injection and discharge, respectively.

\section{Results and Discussion}

3.1. Morphology, Structure, and Composition of the Prepared Nanostructures. The morphology and structure of the nanostructures prepared after thermal treatment of the hydrothermally grown precursors are shown in Figure 1. A snowflake-like morphology is exhibited with roughly six angles in Figure 1(a). Porous nanosheets grow together, while each flake consists of $\sim 6$ to 10 layers of nanosheets, as shown in Figure 1(b) and illustrated by the inset. The structure is well confirmed by TEM images (Figures 1(c) and $1(\mathrm{~d})$ ). Throughout each snowflake, pores in a broad size range distribute densely, forming a three-dimensional network structure, which perhaps provides a good diffusion environment for gas-sensing detection.

The composition of the nanostructures is confirmed by XRD pattern and XPS spectra, as shown in Figures 2 and 3, respectively. In Figure 2, all diffraction peaks can be readily indexed to the hexagonal wurtzite structure of $\mathrm{ZnO}$ crystals, which are in good agreement with the Joint Committee on Powder Diffraction Standards (JCPDS) card (number 361451). For example, the peaks $(2 \theta)$ at about $32.1^{\circ}, 34.8^{\circ}$, and $36.6^{\circ}$ can be attributed to the (100), (002), and (101) crystal planes, respectively. No other diffraction signal was detected, indicating a high purity of the as-synthesized samples. Seen from the XPS survey spectrum of the samples (Figure 3(a)), peaks of $\mathrm{Zn}, \mathrm{O}$, and $\mathrm{C}$ can be observed clearly. The $\mathrm{O}$ peak can be ascribed to both the adsorbed $\mathrm{H}_{2} \mathrm{O}$ and the $\mathrm{O}$ component in $\mathrm{ZnO}$ crystal lattices, while the $\mathrm{C}$ peak is from adsorbed organic species on the surface. In Figure 3(b), the $\mathrm{O}$ spectrum is fitted into three peaks located at about $530.1,531.2,532.3 \mathrm{eV}$, which can be indexed to the $\mathrm{O}^{2-}$ from wurtzite-type $\mathrm{ZnO}$, crystal where $\mathrm{O}$ is insufficient, and the absorbed $\mathrm{H}_{2} \mathrm{O}$, respectively $[17,18]$. In Figure 4(a), it exhibits type IV isotherm with a hysteresis loop from about 0.6 to 1.0 $\left(P / P_{0}\right)$. The BET surface area is about $35.3 \mathrm{~m}^{2} \mathrm{~g}^{-1}$, while the 


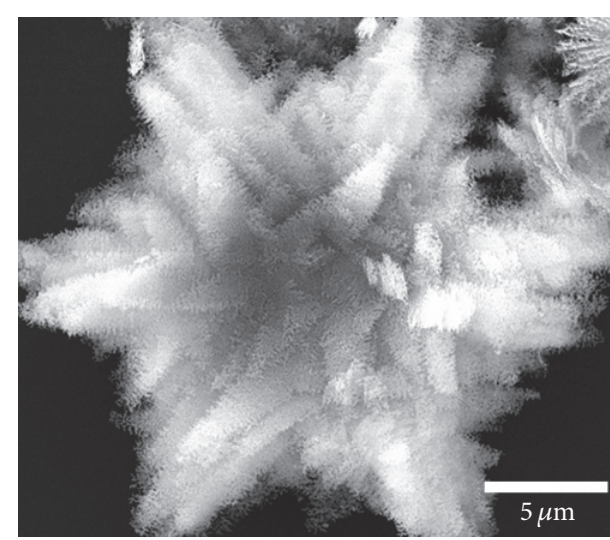

(a)

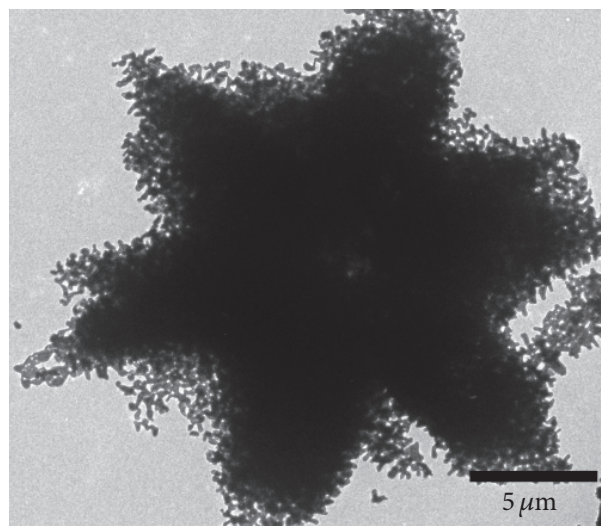

(c)

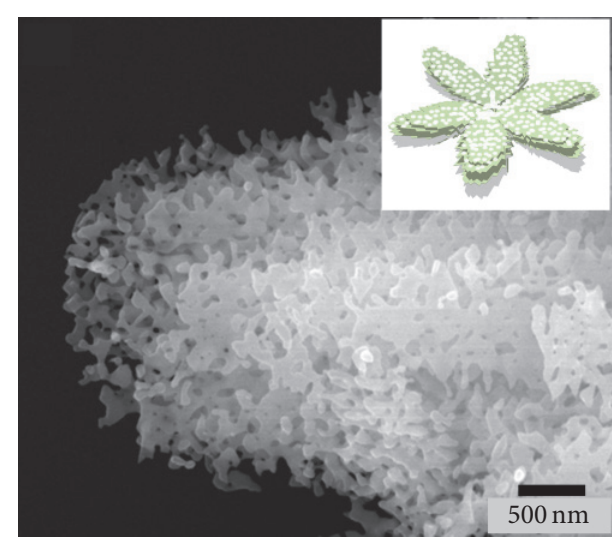

(b)

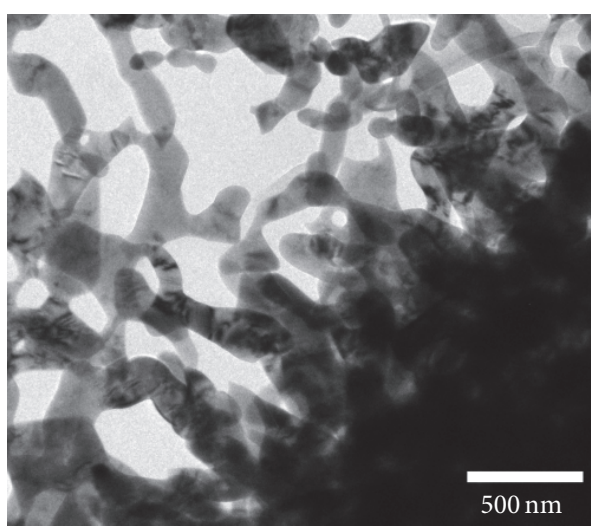

(d)

FIGURE 1: (a) Low- and (b) high-magnification SEM images and (c), (d) TEM photographs of the snowflake-shaped architectures. Inset in (b) is an illustration of the nanostructure.

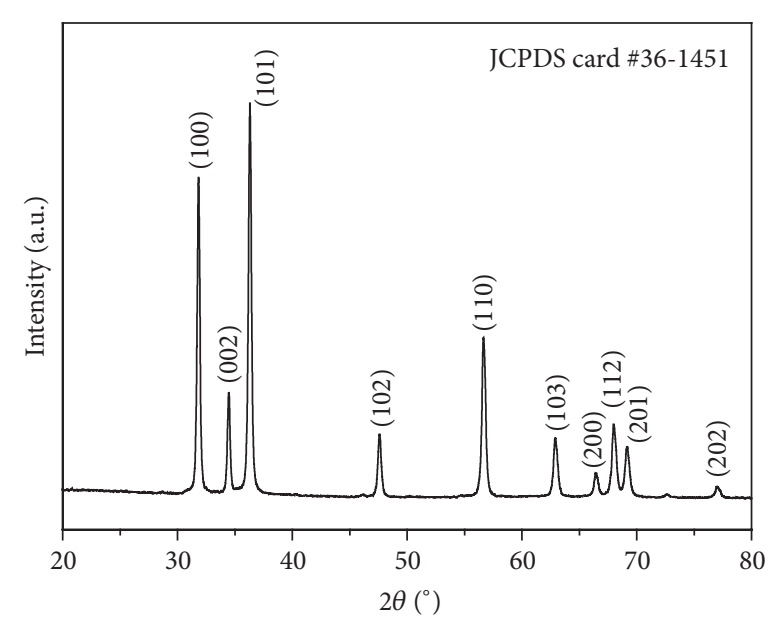

FIGURE 2: XRD pattern of the porous snowflake-shaped $\mathrm{ZnO}$ nanostructures.

pore size distribution was measured using a Barrett-JoynerHalenda $(\mathrm{BJH})$ method (Figure 4(b)) which indicates that the $\mathrm{ZnO}$ nanostructures exhibit both meso- and microporous features. The micropores $(>50 \mathrm{~nm})$ can be ascribed to the pores throughout the snowflake-like nanostructures as confirmed by the TEM images shown above (Figures 1 (c) and $1(\mathrm{~d}))$, while the mesopores $(2 \sim 50 \mathrm{~nm})$ maybe from the interspaces between the multilayered nanoflakes.

3.2. Gas-Sensing Performance. Figure 5 shows the real-time gas-sensing curves of the $\mathrm{ZnO}$ nanostructures-based sensor to diethyl ether, methylbenzene, and ethanol. The current increases when the target gases are injected into the detection chamber; then the current decreases back when the target gases are released by air flow (Figure 5(a)). On the basis of a typical semiconductor sensing mechanism, the adsorption of oxygen in air on the surface of $\mathrm{ZnO}$ produces several ionic species like $\mathrm{O}^{-}, \mathrm{O}^{-2}$, and $\mathrm{O}^{2-}[19,20]$. The electrons in $\mathrm{ZnO}$ are captured, resulting in a high resistance of $\mathrm{ZnO}$ [21]. Once reducing gases such as diethyl ether, methylbenzene, and ethanol in our investigation, contact with $\mathrm{ZnO}$ surface, they will be oxidized by the ionic species, leading to the release of electrons back to $\mathrm{ZnO}$ and thus exhibiting a low resistance.

As we can see in Figure 5, all the measurements to different target gases show similar sensing and recovery behaviors accompanied with current decrease and increase processes. The responses are calculated, as shown in Figure 6(a). It shows that the responses to ethanol are the highest one compared to methylbenzene and diethyl ether. 


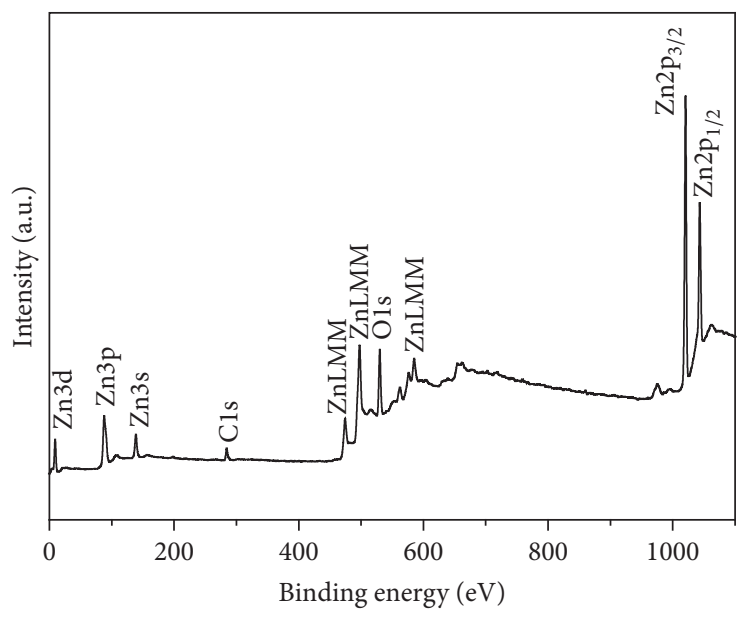

(a)

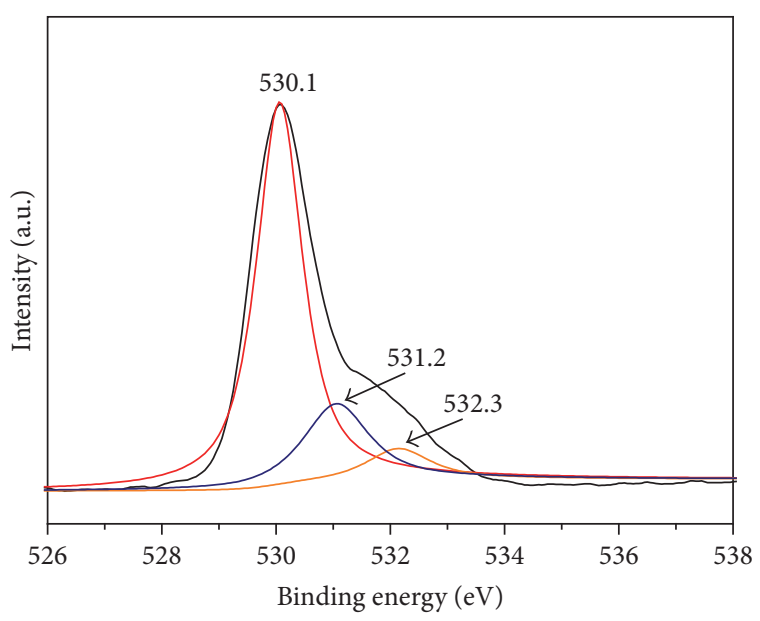

(b)

FIGURE 3: XPS spectra of the snowflake-shaped $\mathrm{ZnO}$ nanostructures: (a) survey spectrum and (b) O $1 \mathrm{~s}$ spectrum.

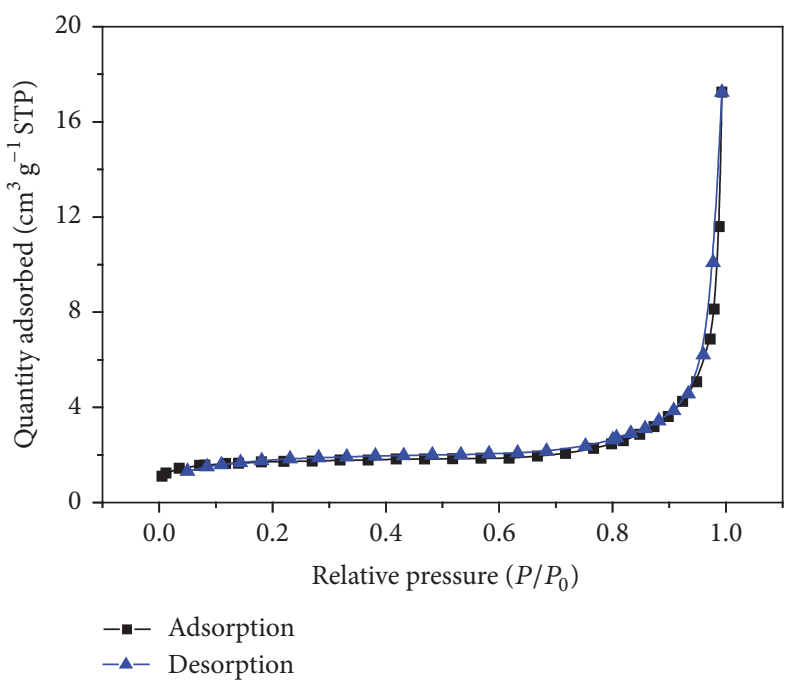

(a)

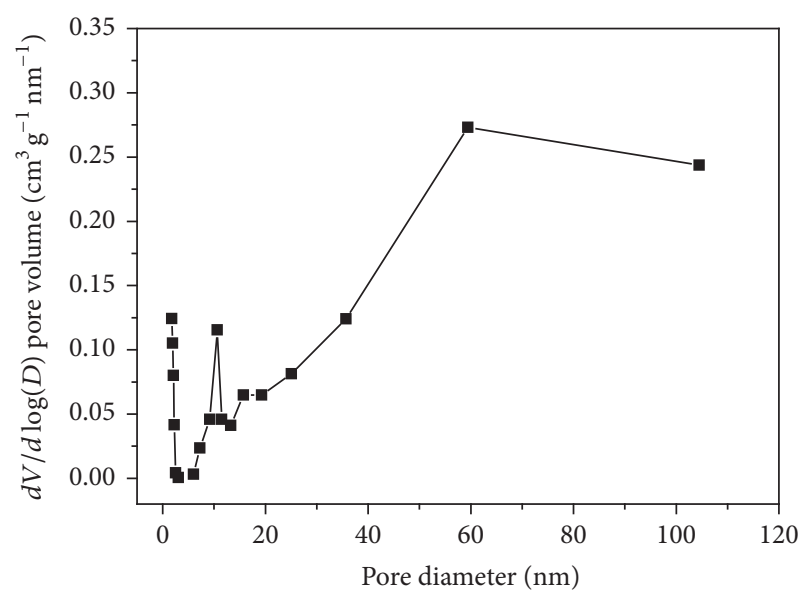

(b)

FIgURE 4: (a) Nitrogen adsorption-desorption isotherm and (b) pore size distribution curve of the porous ZnO nanostructures.

For example, at a gas concentration of $100 \mathrm{ppm}$, the responses to ethanol, methylbenzene, and diethyl ether are about 27, 21, and 11, respectively. The gas-sensing performance is favorably comparable to many other reports $[22,23]$. The response difference perhaps is caused by the different bond energies of target gases, since there are bond breaking and new bond formation processes in gas-sensing reactions between gas molecules and ionic species. In addition, the gas responses exhibit a good linearity of gas concentration versus response, indicating a potentially wide detection range of target gases.

The response and recovery times are shown in Figure 6(b). Both the response and recovery of the present gas sensors are quite rapid. In particular, the response times to diethyl ether and methylbenzene are less than 10 seconds, while the recovery times are about 30 to 50 seconds to ethanol, exhibiting a potential for fast gas detection. In addition, the response time shows a much less change compared to the recovery time. The mechanism could be explained as follows. With a high gas concentration, more small molecular species are produced which adsorb on the surface of $\mathrm{ZnO}$. In this condition, the gas desorption during recovery process becomes difficult, resulting in a long recovery time. For each gas analyte, the formed small molecular species would be different, leading to a different adsorption energy and steric hindrance on the $\mathrm{ZnO}$ sensing layer. The gas adsorption and desorption behaviors during gas-sensing measurements are demonstrated below, which perhaps open a promising new opportunity for specific gas analyte recognition.

As shown in Figure 7, the gas-sensing curves are transformed. Taking the gas-sensing measurements to $50 \mathrm{ppm}$ target gases as an example, the gas-sensing response (i.e., current of gas sensor at here) is transformed from $y$ - to $x$ axis. Meanwhile, the first derivative of response versus time is set as $y$-axis. Under specific conditions, such as temperature, 


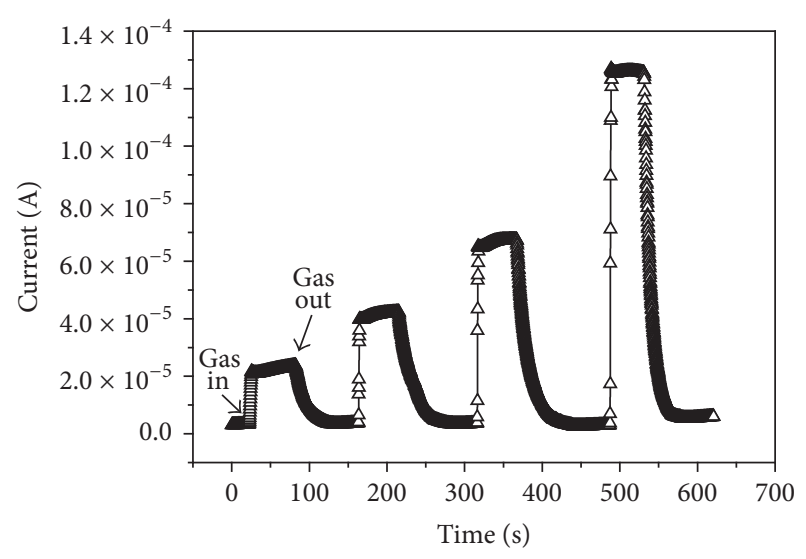

$-\Delta-$ Diethyl ether

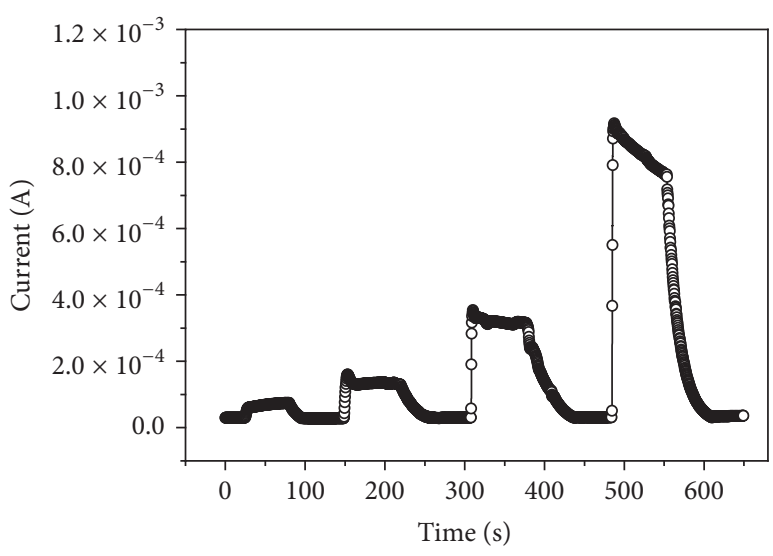

-o- Methylbenzene

(b)

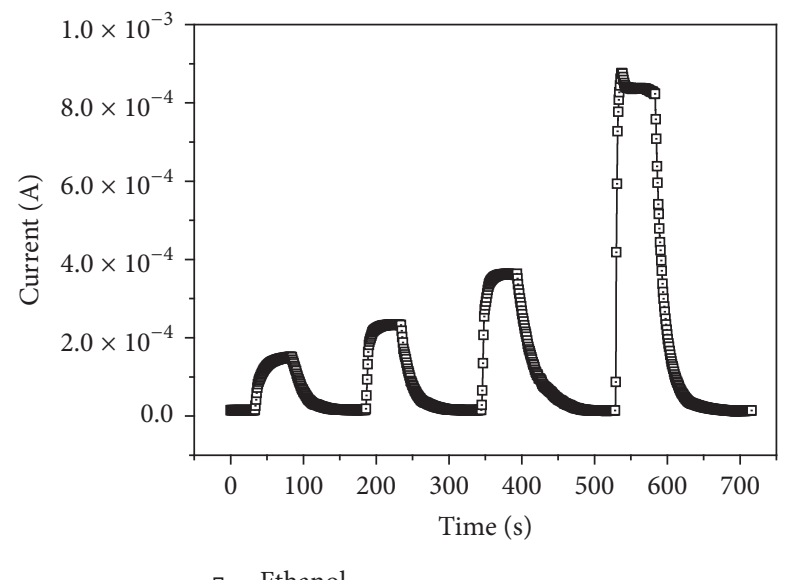

(c)

FIGURE 5: Real-time sensing curves of the snowflake-shaped ZnO-based gas sensor to (a) diethyl ether, (b) methylbenzene, and (c) ethanol. Target gas in/out during measurements was indicated in (a).

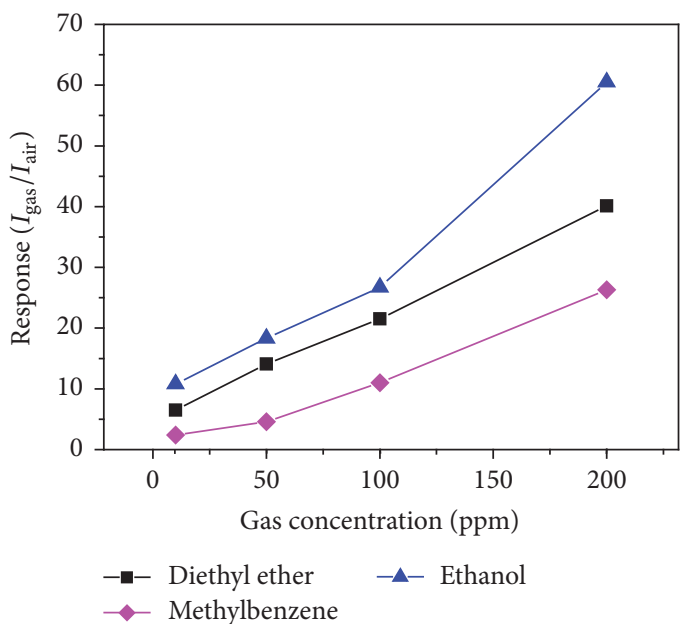

(a)

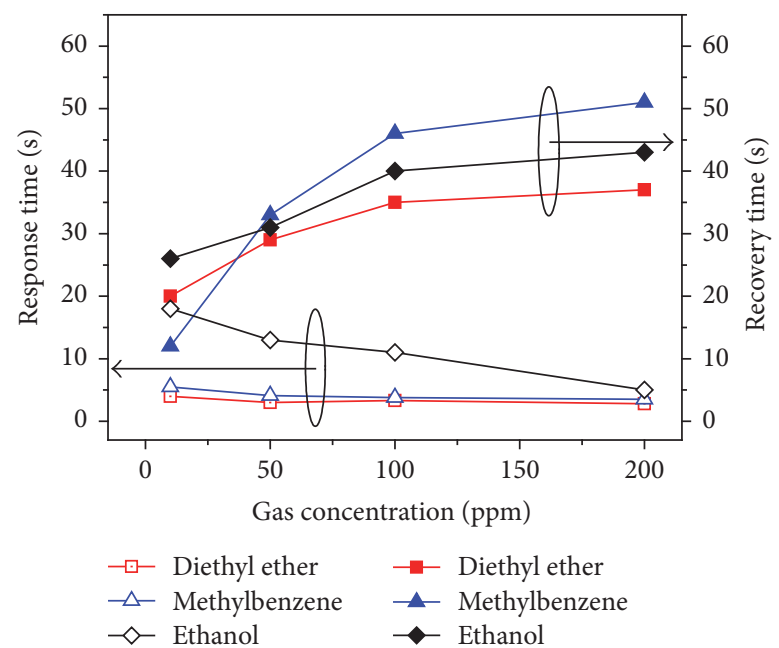

(b)

FIGURE 6: (a) Gas-sensing responses and (b) response and recover times of the $\mathrm{ZnO}$-based gas sensor to target gases. 


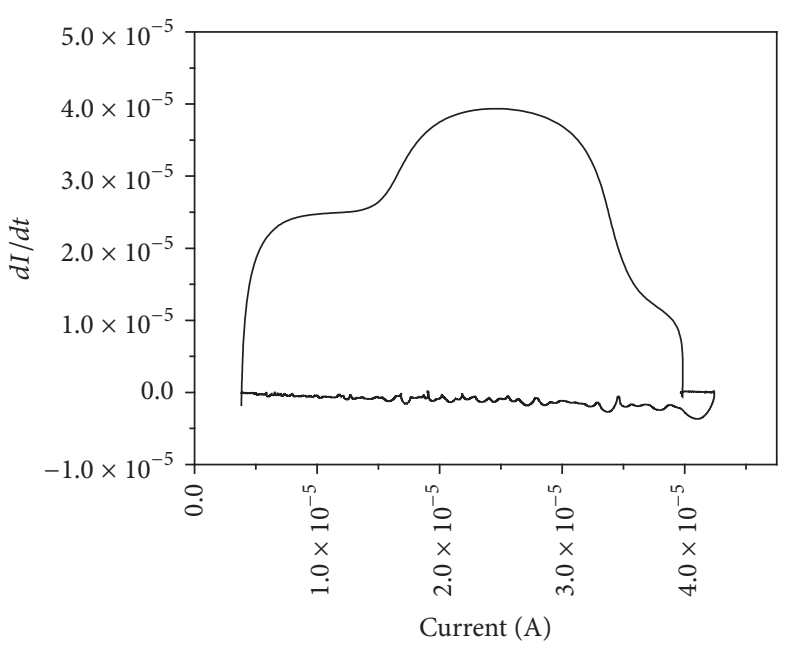

- Diethyl ether

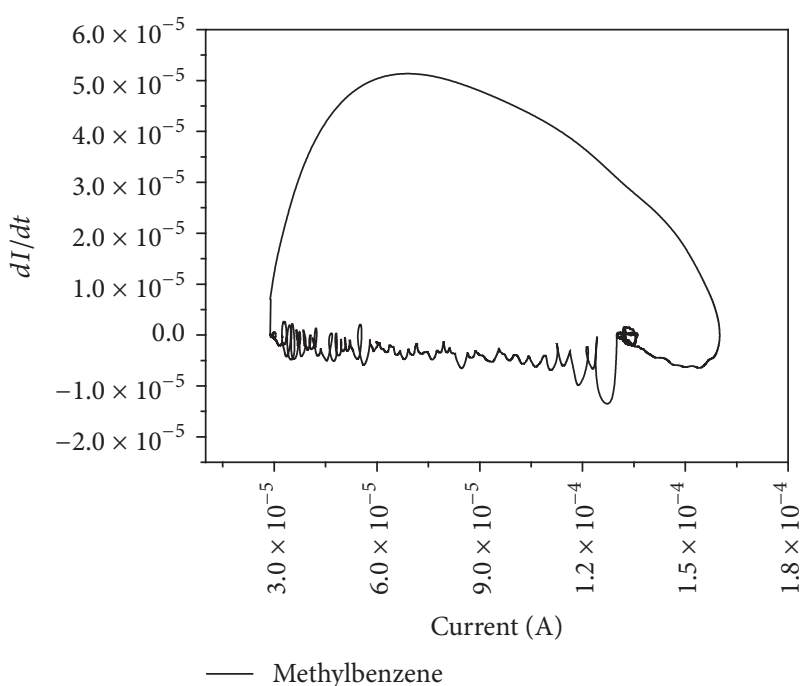

(b)

(a)

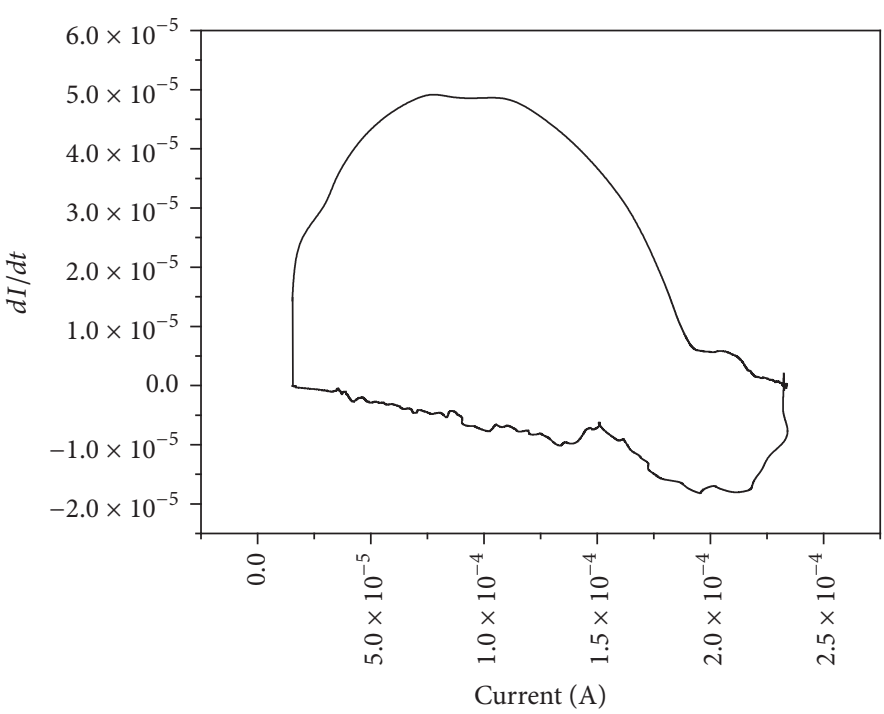

Ethanol

(c)

FIGURE 7: Gas adsorption-desorption curves of the gas sensor based on snowflake-shaped ZnO nanostructures to 50 ppm target gases: (a) diethyl ether, (b) methylbenzene, and (c) ethanol.

surface state of sensing nanomaterials, gas composition, and the resistance of gas sensor are determined [24]. Since that, the current of gas sensor presents the surface state of sensing materials, thus indicating the gas adsorptiondesorption behaviors during gas-sensing detection. As can be seen from Figure 7, in each circuit, the upper part stands for the adsorption, while the under part presents desorption. The relatively smaller area of desorption part of diethyl ether indicates a rapid desorption, which would contribute a short recovery time. It is in agreement with the gassensing measurement results shown above. In addition, all gas adsorption-desorption curves are quite different with each other, suggesting a good potential for practical target recognition accompanying with some automate algorithms.

\section{Conclusions}

In summary, a porous snowflake-shaped $\mathrm{ZnO}$ nanostructure was presented for gas-sensing detection of VOCs including diethyl ether, methylbenzene, and ethanol. The specific hierarchical structure improves the gas diffusion during detection, leading to a high response and short response/recovery time. At a target gas concentration of $100 \mathrm{ppm}$, the responses of the $\mathrm{ZnO}$-based gas sensor to ethanol, methylbenzene, and diethyl ether are about 27, 21, and 11, respectively. The response times to diethyl ether and methylbenzene are less than 10 seconds. In addition, the gas adsorption-desorption kinetics shows that the gas-sensing behaviors to different target gases are quite different, which makes it possible for 
practical gas recognition on the basis of some automate algorithm methods.

\section{Competing Interests}

The authors declare that they have no competing interests.

\section{Authors' Contributions}

Tianli Han, Xuexue Li, and Xiaoman Zhang contribute equally to this work.

\section{Acknowledgments}

This work was financially supported by the "973" State Key Project of Fundamental Research for Nanoscience and Nanotechnology (2011CB933700) and the National Natural Science Foundation of China (51002157, 21277146, 51672176, and 61071054).

\section{References}

[1] N. El Ariebi, J. Hiscox, S. A. Scriven, C. T. Müller, and L. Boddy, "Production and effects of volatile organic compounds during interspecific interactions," Fungal Ecology, vol. 20, pp. 144-154, 2016.

[2] J. Kesselmeier and M. Staudt, "Biogenic volatile organic compounds (VOC): an overview on emission, physiology and ecology," Journal of Atmospheric Chemistry, vol. 33, no. 1, pp. 2388, 1999.

[3] A. Šutka and K. A. Gross, "Spinel ferrite oxide semiconductor gas sensors," Sensors and Actuators, B: Chemical, vol. 222, pp. 95-105, 2016.

[4] L. Kou, T. Frauenheim, and C. Chen, "Phosphorene as a superior gas sensor: selective adsorption and distinct $I-V$ response," Journal of Physical Chemistry Letters, vol. 5, no. 15, pp. 2675-2681, 2014.

[5] A. Sanger, A. Kumar, A. Kumar, and R. Chandra, "Highly sensitive and selective hydrogen gas sensor using sputtered grown Pd decorated $\mathrm{MnO}_{2}$ nanowalls," Sensors and Actuators B: Chemical, vol. 234, pp. 8-14, 2016.

[6] M. A. Arain, M. Trincavelli, M. Cirillo, E. Schaffernicht, and A. J. Lilienthal, "Global coverage measurement planning strategies for mobile robots equipped with a remote gas sensor," Sensors, vol. 15, no. 3, pp. 6845-6871, 2015.

[7] P.-G. Su and L.-Y. Yang, " $\mathrm{NH}_{3}$ gas sensor based on $\mathrm{Pd} / \mathrm{SnO}_{2}$ / RGO ternary composite operated at room-temperature," Sensors and Actuators B: Chemical, vol. 223, pp. 202-208, 2016.

[8] S. Das and V. Jayaraman, " $\mathrm{SnO}_{2}$ : a comprehensive review on structures and gas sensors," Progress in Materials Science, vol. 66, pp. 112-255, 2014.

[9] W. Tang and J. Wang, "Methanol sensing micro-gas sensors of $\mathrm{SnO}_{2}-\mathrm{ZnO}$ nanofibers on $\mathrm{Si} / \mathrm{SiO}_{2} / \mathrm{Ti} / \mathrm{Pt}$ substrate via stepwiseheating electrospinning," Journal of Materials Science, vol. 50, no. 12, pp. 4209-4220, 2015.

[10] S. Sharma, S. Hussain, S. Singh, and S. S. Islam, "MWCNTconducting polymer composite based ammonia gas sensors: a new approach for complete recovery process," Sensors and Actuators, B: Chemical, vol. 194, pp. 213-219, 2014.
[11] B. C. Yadav, K. Agrahari, S. Singh, and T. P. Yadav, "Fabrication and characterization of nanostructured indium tin oxide film and its application as humidity and gas sensors," Journal of Materials Science: Materials in Electronics, vol. 27, no. 5, pp. 4172-4179, 2016.

[12] H.-J. Kim and J.-H. Lee, "Highly sensitive and selective gas sensors using p-type oxide semiconductors: overview," Sensors and Actuators, B: Chemical, vol. 192, pp. 607-627, 2014.

[13] S. S. Varghese, S. Lonkar, K. K. Singh, S. Swaminathan, and A. Abdala, "Recent advances in graphene based gas sensors," Sensors and Actuators B: Chemical, vol. 218, pp. 160-183, 2015.

[14] S. Nasirian and H. M. Moghaddam, "Polyaniline assisted by $\mathrm{TiO}_{2}: \mathrm{SnO}_{2}$ nanoparticles as a hydrogen gas sensor at environmental conditions," Applied Surface Science, vol. 328, pp. 395404, 2015.

[15] X. Zhang, J. Liu, S. J. Kelly, X. Huang, and J. Liu, "Biomimetic snowflake-shaped magnetic micro-/nanostructures for highly efficient adsorption of heavy metal ions and organic pollutants from aqueous solution," Journal of Materials Chemistry A, vol. 2, no. 30, pp. 11759-11767, 2014.

[16] F. Meng, N. Hou, Z. Jin et al., "Ag-decorated ultra-thin porous single-crystalline $\mathrm{ZnO}$ nanosheets prepared by sunlight induced solvent reduction and their highly sensitive detection of ethanol," Sensors and Actuators B: Chemical, vol. 209, pp. 975982, 2015.

[17] K. Mageshwari, D. Nataraj, T. Pal, R. Sathyamoorthy, and J. Park, "Improved photocatalytic activity of $\mathrm{ZnO}$ coupled $\mathrm{CuO}$ nanocomposites synthesized by reflux condensation method," Journal of Alloys and Compounds, vol. 625, pp. 362-370, 2015.

[18] H. Kung and A. Teplyakov, "Selectivity and mechanism of thermal decomposition of $\beta$-diketones on $\mathrm{ZnO}$ powder," Journal of Catalysis, vol. 330, pp. 145-153, 2015.

[19] C.-J. Chang, C.-Y. Lin, J.-K. Chen, and M.-H. Hsu, "Ce-doped $\mathrm{ZnO}$ nanorods based low operation temperature $\mathrm{NO}_{2}$ gas sensors," Ceramics International, vol. 40, no. 7, pp. 10867-10875, 2014.

[20] Z. S. Hosseini, A. Mortezaali, A. I. Zad, and S. Fardindoost, "Sensitive and selective room temperature $\mathrm{H}_{2} \mathrm{~S}$ gas sensor based on Au sensitized vertical $\mathrm{ZnO}$ nanorods with flower-like structures," Journal of Alloys and Compounds, vol. 628, pp. 222229, 2015.

[21] X. Pan, X. Zhao, J. Chen, A. Bermak, and Z. Fan, "A fastresponse/recovery $\mathrm{ZnO}$ hierarchical nanostructure based gas sensor with ultra-high room-temperature output response," Sensors and Actuators B: Chemical, vol. 206, pp. 764-771, 2015.

[22] S. Nag, A. Sachan, M. Castro, V. Choudhary, and J.-F. Feller, "Spray layer-by-layer assembly of POSS functionalized CNT quantum chemo-resistive sensors with tuneable selectivity and ppm resolution to VOC biomarkers," Sensors and Actuators, B: Chemical, vol. 222, pp. 362-373, 2016.

[23] A. Urrutia, J. Goicoechea, and F. J. Arregui, "Optical fiber sensors based on nanoparticle-embedded coatings," Journal of Sensors, vol. 2015, Article ID 805053, 18 pages, 2015.

[24] J. Liu, F. Meng, T. Luo, W. Li, M. Li, and J. Liu, "Novel facile detection of persistent organic pollutants using highly sensitive gas sensor," Talanta, vol. 82, no. 1, pp. 409-416, 2010. 

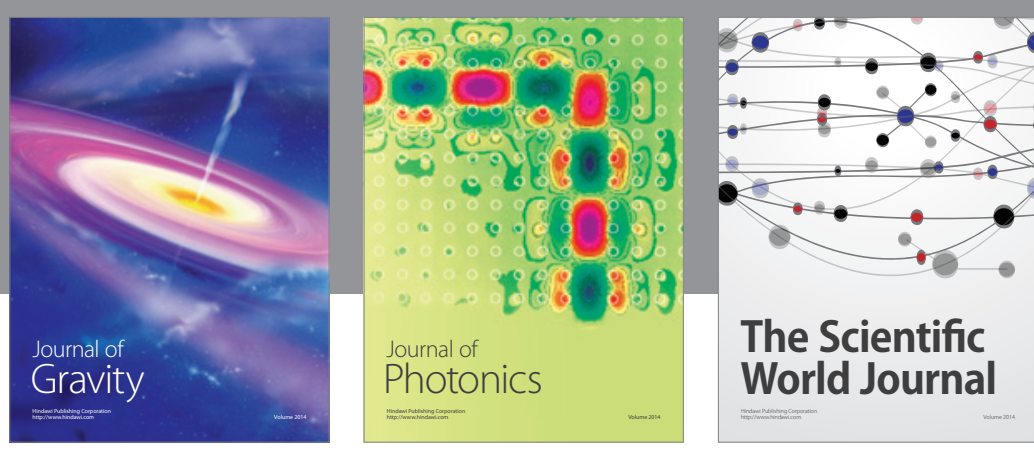

The Scientific World Journal
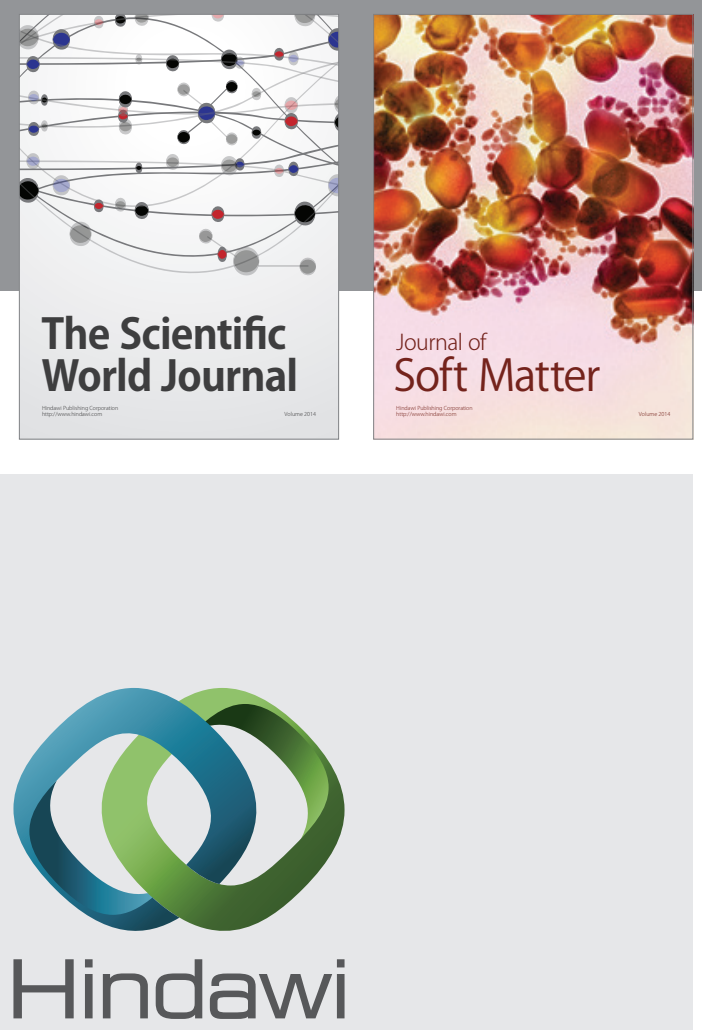

Submit your manuscripts at

https://www.hindawi.com
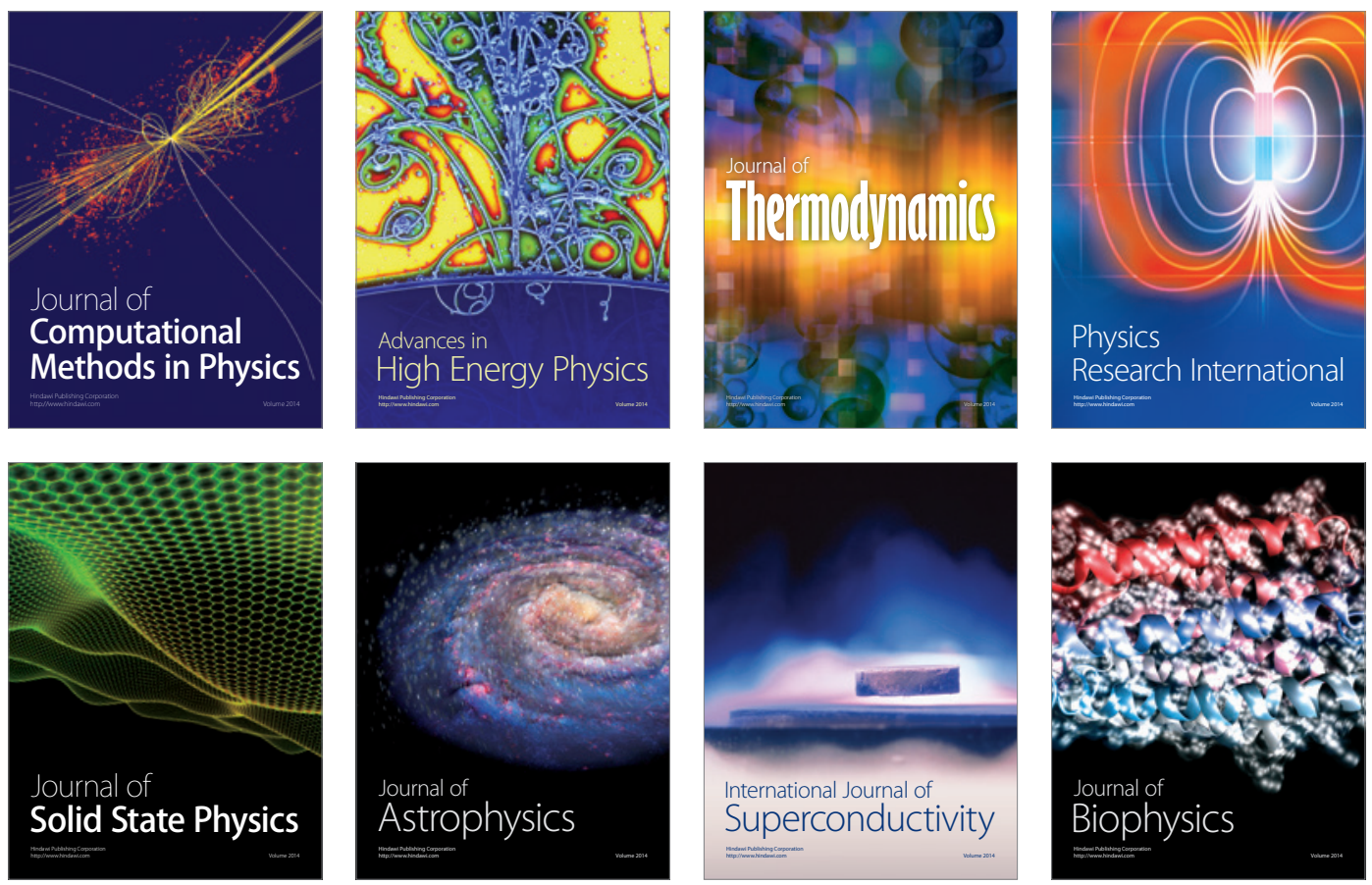
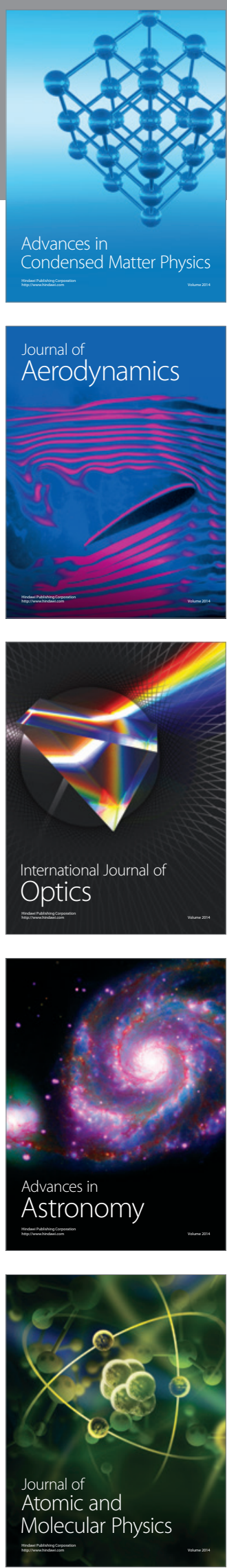